\title{
"Delayed Pial Vessels" in Multiphase CT Angiography Aid in the Detection of Arterial Occlusion in Anterior Circulation
}

W e read the article by Byrne et al, "Improved Detection of Anterior Circulation Occlusions: The 'Delayed Vessel Sign' on Multiphase CT Angiography” with great interest. ${ }^{1}$ However, we would like to point out other important aspects of multiphase CT angiography (mCTA) to the readers of the American Journal of Neuroradiology. Multiphase CTA is a useful recent addition to neurovascular imaging in acute stroke, providing information on the degree and extent of pial arterial filling in the whole brain in a time-resolved manner. ${ }^{2}$ It was designed to assess pial vessel asymmetry in terms of delayed contrast filling and/or delayed contrast washout compared with the contralateral side. The time-resolved imaging acquisition additionally enables judgment on either antegrade or retrograde pial vessel contrast filling and thus gives the reader information on clot permeability, collateral circulation, and possible response to thrombolytic therapy.

A recent study from our group was able to show an increase in the detection of anterior circulation intracranial occlusion with mCTA, especially for distal occlusions. ${ }^{3}$ In the presence or absence of obvious large-vessel occlusion, pial vessel asymmetry in the second and third phase added to the yield of mCTA for occlusion detection. The "delayed vessel sign" mentioned in the study by Byrne et $\mathrm{al}^{1}$ is one but not the only component of pial vessel asymmetry as explained above. Both low-flow (upstream stenoocclusive lesions) and high-flow (recanalization) states could cause pial vessel asymmetry as proposed in our article. ${ }^{3}$

Our study also established that mCTA is a user-friendly imaging tool that can improve the diagnostic accuracy for anterior

http://dx.doi.org/10.3174/ajnr.A5529 circulation occlusions, even when used by relatively inexperienced readers and when occlusions are more distal. Thus, inclusion of multiphase CTA in acute ischemic stroke imaging protocols has the potential to allow non-radiology trained physicians and trainees to rapidly and accurately determine anterior circulation occlusions, a crucial point in clinical management when considering patients for revascularization procedures. ${ }^{3}$ Furthermore, pial vessel dynamics captured on mCTA can also be quantified to provide information similar to that of perfusion CT and can be used to predict tissue fate regionally in patients with acute ischemic stroke. ${ }^{4}$

\section{REFERENCES}

1. Byrne D, Sugrue G, Stanley E, et al. Improved detection of anterior circulation occlusions: the "delayed vessel sign" on multiphase CT angiography. AJNR Am J Neuroradiol 2017;38:1911-16 CrossRef Medline

2. Menon BK, d'Esterre CD, Qazi EM, et al. Multiphase CT angiography: a new tool for the imaging triage of patients with acute ischemic stroke. Radiology 2015;275:510-20 CrossRef Medline

3. Yu AY, Zerna C, Assis Z, et al. Multiphase CT angiography increases detection of anterior circulation intracranial occlusion. Neurology 2016;87:609-16 CrossRef Medline

4. d'Esterre CD, Trivedi A, Pordeli P, et al. Regional comparison of multiphase computed tomographic angiography and computed tomographic perfusion for prediction of tissue fate in ischemic stroke. Stroke 2017;48:939-45 CrossRef Medline

(1) R.-J. Singh

(D). Zerna

(1)B.K. Menon

Calgary Stroke Program, Department of Clinical Neuroscience University of Calgary Calgary, Alberta, Canada 